\title{
AGRONOMIC TRAITS OF DUAL-PURPOSE WHEAT WITH DIFFERENT PLANT ARCHITECTURES UNDER DEFOLIATION STRATEGIES
}

\author{
CARACTERÍSTICAS AGRONÔMICAS DE TRIGO DUPLO-PROPÓSITO COM \\ DIFERENTES ARQUITETURAS DE PLANTAS SOB ESTRATÉGIAS DE \\ DESFOLHAÇÃO
}

\author{
Giselle Regina RODOLFO'; Clovis Arruda SOUZA²; André Fischer SBRISSIA²; \\ Deivid Luis Vieira STEFEN ${ }^{1}$ \\ 1. Doutores em Produção Vegetal, Universidade do Estado de Santa Catarina - UDESC, Lages, SC, Brasil; \\ 2. Professores, Doutores, Universidade do Estado de Santa Catarina - UDESC, Lages, SC, Brasil. souza_clovis@yahoo.com.br
}

\begin{abstract}
Crop-livestock system implies in the balance of appropriate animal foraging, minimizing effects on grain production on the same crop season. This study aims to assess canopy heights $(20$ and $30 \mathrm{~cm})$ and cuts number (no cut, 1, 2 and 3 cuts) effects on wheat productivity and bromatological composition of forage, and grain yield of BRS Umbu and BRS Tarumã cultivars. Regardless cuts number and cultivars, $30 \mathrm{~cm}$ provided higher forage productivity (1032 and $\left.1348 \mathrm{~kg} \mathrm{DM} \mathrm{ha}^{-1}\right)$ than $20 \mathrm{~cm}\left(554\right.$ and $\left.909 \mathrm{~kg} \mathrm{DM} \mathrm{ha}{ }^{-1}\right)$, for BRS Umbu and Tarumã, respectively, in average crop seasons. For both cultivars and crop seasons, mean values of $26 \%$ crude protein, $55 \%$ neutral detergent fiber and $26 \%$ acid detergent fiber were obtained. At $20 \mathrm{~cm}$ no difference in grain yield was observed as a function of cuts number with $3780 \mathrm{~kg} \mathrm{ha}^{-1}$ for BRS Umbu, in average crop seasons and $4306 \mathrm{~kg} \mathrm{ha}^{-1}$ in 2014, for BRS Tarumã. However, at $30 \mathrm{~cm}$, the grain yield was penalized at least in $30 \%$ as a result only third cut. These results indicate that wheat as dual-purpose can be employed in southern Brazil in grazing systems in forage shortage times and then produce grains without yield penalties as a function to the management used.
\end{abstract}

KEYWORDS: Triticum aestivum L. Integrated crop livestock system. Management height. Cuts number. Grain yield.

\section{INTRODUCTION}

In southern Brazil one of the biggest limitations to livestock farming is forage lack in period from fall to early winter; a time when the summer forage species reduced productivity, and winter forage species are still not satisfactorily developed. In addition to traditionally alternatives used in critical period, such as annual ryegrass and oat pastures, silage and hay, dual-purpose cereals like wheat can provide forage to animals and grains in same crop (MARIANI et al., 2012). Moreover, wheat is considered a forage with good nutritional quality, when grazed on vegetative growth (HARRISON et al., 2011; HARRISON et al., 2014).

Wheat cultivars differ in some traits such as tiller emission number, plant architecture, cycle length and consequently the productive potential (SANGOI et al., 2007). Plant architecture with erect growth habit improves the light distribution, canopy light use efficiency, and biomass production, mainly reducing the self-shading among leaves to allow more radiation to penetrate into the lower parts of canopies (LI et al., 2016; SAKAMOTO et al., 2006). Among brazilian dual-purpose wheat cultivars (grains and animal fodder), stands out, with plant architecture very distinct, BRS Umbu which shows erect leaves habit and BRS Tarumã that presents prostrate leaves habit. BRS Umbu, potentially targets for cutting-hay and silage, large dry matter productivity, which reaches up 10 tons $\mathrm{ha}^{-1}$. Further, the spike in this cultivar lack awns, and thus do not cause irritation for esophageal mucosa (LEHMEN et al., 2014). This cultivar also displays high grain yield, reaching 3.47 tons $\mathrm{ha}^{-1}$ (MEINERZ et al., 2011), which is of interest as this plant fraction has great energy content. Moreover, high crude protein (CP) concentrations (up $83 \mathrm{~g} \mathrm{~kg}^{-1}$ dry matter) have been reported in BRS Umbu silage (LEHMEN et al., 2014). BRS Tarumã cultivar with prostrate habit and smaller leaf blades, has a greater potential for direct grazing, with accumulated forage production (DM) of 7.42 tons $\mathrm{ha}^{-1}$ (TAFFAREL et al., 2017); grain yield of 3.54 tons.ha $^{-1}$ (HENZ et al., 2016), presents a long vegetative cycle (ZANON et al., 2012) and forage crude protein around $195.6 \mathrm{~g}$ $\mathrm{kg}^{-1}$ DM. Further, the spikes in this cultivar is awneds. Due to awns presence, this cultivar is not recommended for silage production. 
Management strategies in wheat cultivars with different growth habits and development cycle influence canopy structure, forage, and grains (BUTCHEE; EDWARDS, 2013). In most international works carried out with dual-purpose winter cereals, phenological stage predominates as a management tool, suggesting that cuts should be made up to when the stem starts to elongate, until growth stage code 30 (GS 30) (ZADOKS et al., 1974). However, there are some recommendations that these crops can be grazed or cut when plants reach $30 \mathrm{~cm}$ height (FONTANELI et al., 2009; HASTENPFLUG et al., 2011a; MARTIN et al., 2013; MEINERZ et al., 2012). Control of defoliation intensity avoids damage to apical meristem, and keep source-sink relationship equilibrium by shoot remove, enabling grain production (MARTIN et al., 2010; PEREIRA et al., 2012). Thus, it is important to consider the intensity and cuts number in dual-purpose cereal production system.

Dual-purpose cultivars used in southern Brazil is mainly concentrated in some research projects in Rio Grande do Sul, Paraná and recently in Santa Catarina states. Due to lack of information and management tools complexity, it is necessary a refinement of management recommendations for dual-purpose wheat cultivars with different growth habits, highlighting the use of more practical management tools, such as plant height. This study aims to assess canopy heights and cuts number effects on wheat agronomic characteristics such as forage productivity, bromatological composition, and grain yield of BRS Umbu and BRS Tarumã cultivars.

\section{MATERIALS AND METHODS}

Experiments were conducted in Lages, Santa Catarina state, southern Brazil, from May (seeding day 5) to November (harvest day 26) in 2013 and 2014 with wheat BRS Umbu cultivar; and same period in 2014 and 2015 with BRS Tarumã cultivar. Wheat cultivars have different characters: BRS Umbu has medium late cycle and cespitoseerect growth habit and BRS Tarumã has late cycle and cespitose-prostrate habit.

Experimental area soil was classified as loamy Haplumbrept (EMBRAPA, 2013), and according to analysis, ( 0 to $20 \mathrm{~cm}$ layer) soil $\mathrm{pH}_{\text {(water) }}$, organic matter, Mehlich-1 P, Mehlich-1 K, $\mathrm{Ca}, \mathrm{Mg}, \mathrm{Al}$ and $\mathrm{H}+\mathrm{Al}$ were, respectively, 5.6, $33 \mathrm{~g}$ $\mathrm{kg}^{-1}, 8.3 \mathrm{mg} \mathrm{dm}^{-3}, 0.47 \mathrm{cmol}_{\mathrm{c}} \mathrm{dm}^{-3}, 4.5 \mathrm{cmol}_{\mathrm{c}} \mathrm{dm}^{-3}$, $2.4 \mathrm{cmol}_{\mathrm{c}} \mathrm{dm}^{-3}, 0.7 \mathrm{cmol}_{\mathrm{c}} \mathrm{dm}^{-3}$ and $9.9 \mathrm{cmol}_{\mathrm{c}} \mathrm{dm}^{-3}$. Weather data for experimental period were obtained and provided by Weather Station from Rural Extension and Agricultural Research of Santa Catarina (INMET / EPAGRI / CIRAM) (Figure 1).

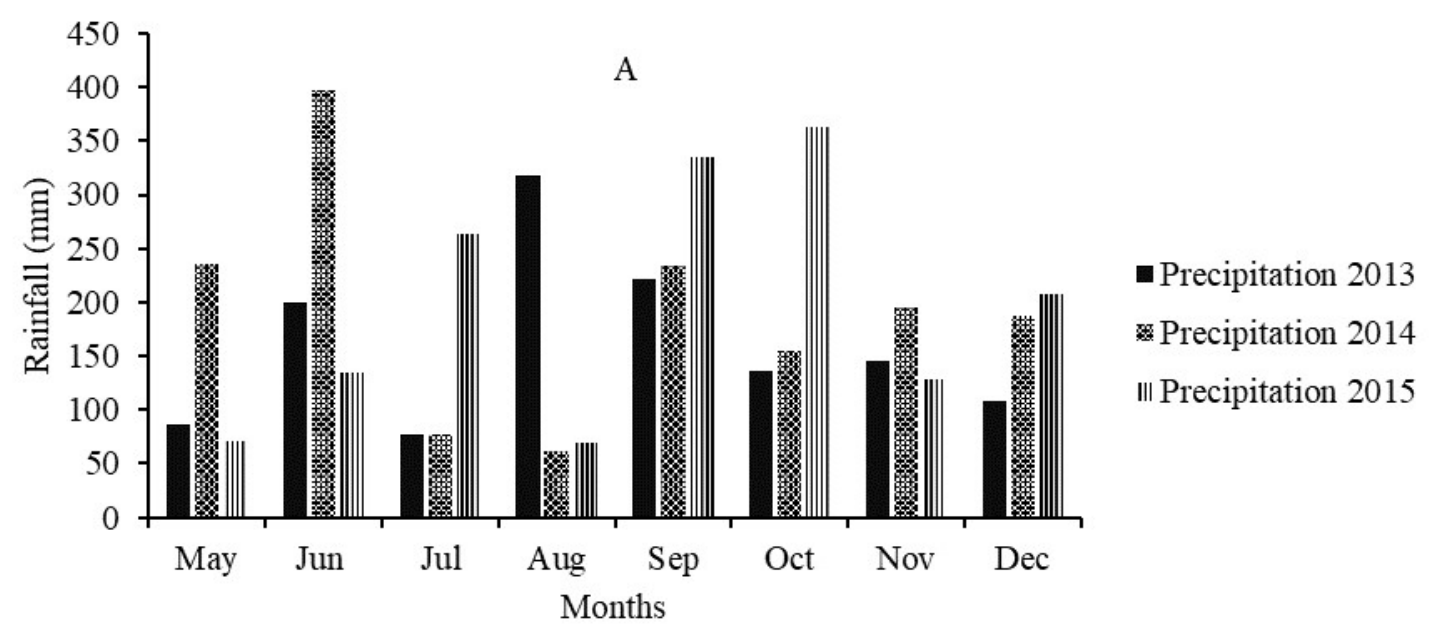




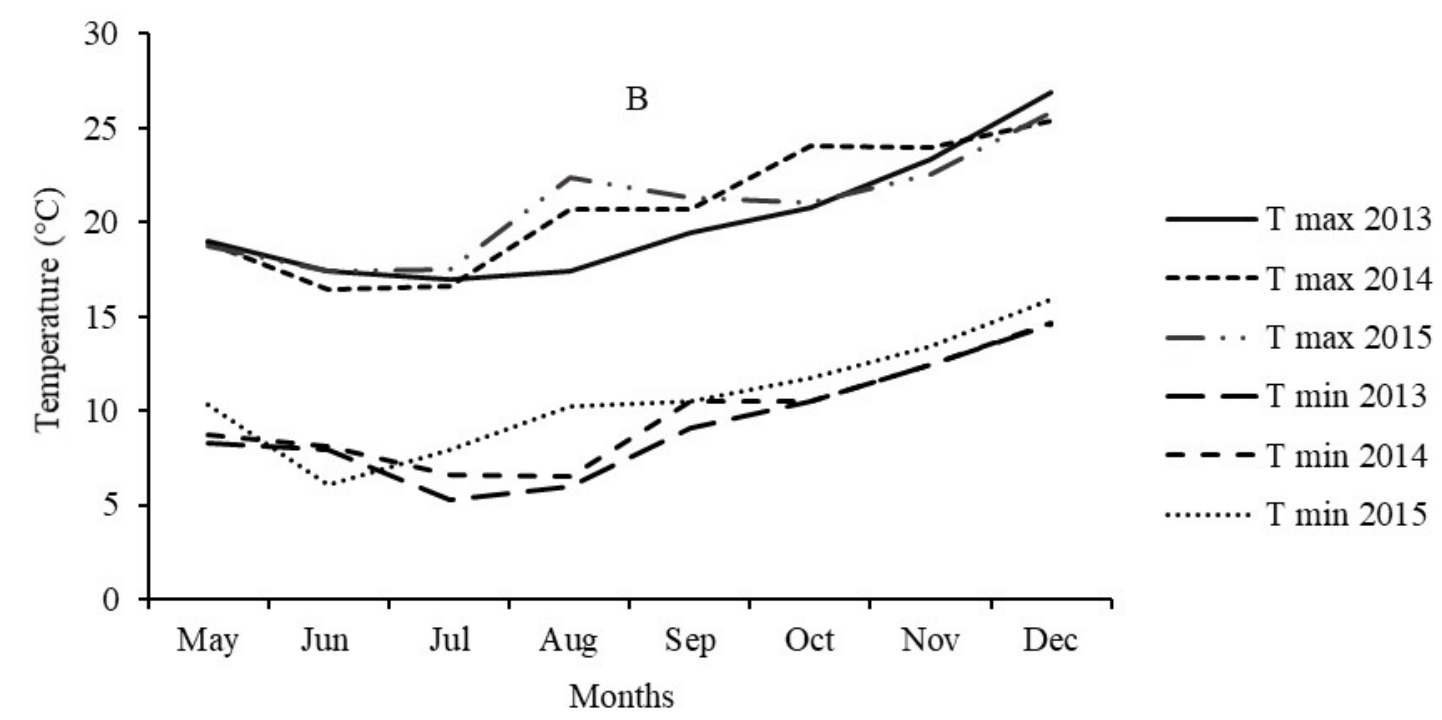

Figure 1. Climatic data of accumulated monthly precipitation (A) and average monthly temperatures (B) in the experimental period, in Lages, SC.

Before first trial year, in 2013, the area was made up by rangeland. Preparation was made with application of 7 tons of dolomitic limestone accompanied by plowing and harrowing. In 2014 and 2015 wheat was sown under no-tillage in common bean and soybean straw, respectively. Was used mineral fertilizer $\mathrm{N}-\mathrm{P}_{2} \mathrm{O}_{5}-\mathrm{K}_{2} \mathrm{O}$ in formulation $5-20-10(\%)$ at a rate of $400 \mathrm{~kg} \mathrm{ha}^{-1}$, per crop season. Nitrogen topdressing from urea was applied with 50 $\mathrm{kg} \mathrm{ha}^{-1} \mathrm{~N}$, at tillering stage (GS 21), at first visible node (GS 31), and after each cut as a replacement harvested nitrogen removal. When replacement fertilization coincided with the fertilization of GS 31 stage, $\mathrm{N}$ fertilization was the same, that is, performed only once.

Seeds were treated with fungicide and insecticide suitable for culture: carbendazim (Methyl benzimidazol-2-ylcarbamate), imidacloprid [1-(6-chloro-3-pyridylmethyl)-N-nitroimidazolidin2-yilideneamine] and, tiodicarb (3,7,9,13tetramethyl-5,11-dioxa-2,8,14-trithia-4,7,9,12-tetraazapentadeca-3,12-diene-6,10-dione) and sowings were made with a plot seeder, where each plot was made up of five rows spaced $20 \mathrm{~cm}$ and seeds deposited at 2-5 cm depth. Density of seeding was 350 seeds per square meter (FONTANELI et al., 2012). Size of each plot was $6 \mathrm{~m}^{2}$ and experiment was composed by 32 plots of each cultivar. For insecticide and fungicide applications the same recommendations of traditional grain production were followed.

Wheat plant height before the defoliation was the criterion for cuts, thus two contrasting canopy heights were stubble residual height (FONTANELI et al., 2009; HASTENPFLUG et al., 2011a; MARTIN et al., 2013; MEINERZ et al., 2012). For defoliation intensity, was used $50 \%$ of canopy height, based on intensities used by Mezzalira et al. (2014) for black oat (Avena strigosa Schreb.). Similar to Carletto et al. (2015) and Meinerz et al. (2012), three successive cuts were made in plants regrowth.

Treatments consisted in a combination of canopy heights (20 and $30 \mathrm{~cm}$ ) and cuts number (no cut, 1, 2, and 3 ), resulting in treatments: 20/1, 20/2, $20 / 3,30 / 1,30 / 2, \quad 30 / 3$ and uncut control. A randomized complete block design was used and treatments were distributed in a $2 \times 4$ factorial arrangement (heights and cuts number), with four replications. Each cultivar was considered an independent experiment, analyzed separately.

Canopy height was monitored weekly, using a graduated sward stick (BARTHRAM, 1985) at 30 random points per plot and plants phenologic stage subjected to each treatment, was evaluated using the Zadoks scale (ZADOKS et al., 1974). When plants achieved 20 or $30 \mathrm{~cm}$, before each cut, tiller population density (TPD) was evaluated by tillers counting contained within a $50 \mathrm{~cm}$ x $50 \mathrm{~cm}$ frame placed in three central lines of plot. In uncut plots, tillers counting were performed at GS 31, thus ensuring that tillering had ceased. Growth stage code 31 was recognized when first plant node at basal region was visible.

Upon achieving 20 and $30 \mathrm{~cm}$, plants were cut using scissors to $50 \%$ of canopy height, corresponding to 10 and $15 \mathrm{~cm}$ stubble height. A $0.5 \mathrm{~m}$ edge was discarded from each end of plot. All shoot material from three central lines was cut and collected. Then, shoot material was dried in forced 
air at $60^{\circ} \mathrm{C}$ to constant weight, from which the forage production was calculated.

Plant materials were ground to pass for analyses of crude protein (CP) according to AOAC (1990), neutral detergent fiber (NDF) and acid detergent fiber (ADF) according to Van Soest et al. (1991). Crude protein was determined by micro Kjeldahl method, multiplying the nitrogen concentration value by 6.25 . Neutral detergent fiber and ADF analyzes were performed sequentially with adaptations for using Fiber Analyzer device (ANKOM). Crude protein, NDF and, ADF levels were expressed in relation to dry matter (DM) (\%).

With plants regrowth and grain production, before harvest, spikes were collected in 1 linear meter of central line of each plot and later estimated the spikes number per square meter. Plants height (H) was determined with a rule graduated in centimeters. Grain was harvested when plants reached harvest maturity, with a combine plot (Wintersteiger).

After harvest, spikelets per spike number were quantified (NSS) and grains per spike number (NGS) from the spikes collected from each plot.
Thousand grain weight (TGW) was determined by counting one thousand grains from each experimental plot, with an automatic grain counter (Sanick model ESC 2011) and subsequent weighing. Grain yield (GY) was determined from production of plots useful area, corrected at $13 \%$ standard moisture.

For statistical analysis, crop seasons and cultivars were not considered as factors. This because are different habits and growth cycles and the intention was obtaining results by different plants conditions. Crop seasons are used for data confiability, and no comparisons in time. Thus, data were submitted to analysis of variance (ANOVA) and when a significant $F$-test was observed, means were compared by Tukey test at 5\% probability using the statistical SAS ${ }^{\circledR}$ program version 9.0.

\section{RESULTS AND DISCUSSION}

Tables 1 and 2 refers to dates and days after sowing at which cuts were performed, plant phenological stage at time of cuts and anthesis dates. This data helps explain the results obtained.

Table 1. Days of cuts, days after sowing (DAS) and respectives phenological stages of dual-purpose wheat BRS Umbu and BRS Tarumã cultivars, in two crop seasons.

\begin{tabular}{|c|c|c|c|c|c|c|}
\hline \multirow[b]{3}{*}{ Cuts } & \multicolumn{3}{|c|}{$20 \mathrm{~cm}$} & \multicolumn{3}{|c|}{$30 \mathrm{~cm}$} \\
\hline & Date & DAS & Stage & Date & DAS & Stage \\
\hline & \multicolumn{6}{|c|}{ BRS Umbu } \\
\hline \multicolumn{7}{|l|}{ Year 2013} \\
\hline First & July, 2 & 57 & 23 & July, 15 & 70 & 25 \\
\hline Second & July, 15 & 70 & 25 & August, 5 & 91 & 31 \\
\hline Third & July, 29 & 84 & 31 & August, 19 & 105 & 33 \\
\hline \multicolumn{7}{|l|}{ Year 2014} \\
\hline First & July, 8 & 50 & 23 & July, 21 & 63 & 25 \\
\hline Second & July, 21 & 63 & 25 & August, 4 & 77 & 31 \\
\hline Third & July, 28 & 70 & 27 & August, 12 & 85 & 32 \\
\hline & \multicolumn{6}{|c|}{ BRS Tarumã } \\
\hline \multicolumn{7}{|l|}{ Year 2014} \\
\hline First & July, 14 & 56 & 23 & August, 18 & 91 & 28 \\
\hline Second & August, 5 & 78 & 25 & September, 1 & 104 & 32 \\
\hline Third & August, 12 & 85 & 27 & September, 8 & 111 & 33 \\
\hline \multicolumn{7}{|l|}{ Year 2015} \\
\hline First & July, 19 & 73 & 25 & August, 4 & 89 & 28 \\
\hline Second & August, 3 & 88 & 28 & August, 15 & 100 & 31 \\
\hline Third & August, 11 & 96 & 31 & August, 24 & 109 & 33 \\
\hline
\end{tabular}


Table 2. Dates of dual-purpose anthesis of BRS Umbu and BRS Tarumã cultivars as a funtion of pre-cut height and cuts number, in two crop seasons.

\begin{tabular}{lcccc}
\hline & \multicolumn{4}{c}{ Anthesis dates } \\
\cline { 2 - 5 } Treatment & BRS Umbu - 2013 & BRS Umbu - 2014 & BRS Tarumã - 2014 & BRS Tarumã - 2015 \\
\hline Control & September, 9 & September, 16 & September, 22 & September, 20 \\
$20 \mathrm{~cm} / 1$ cut & September, 9 & September, 16 & September, 22 & September, 20 \\
$20 \mathrm{~cm} / 2$ cuts & September, 16 & September, 19 & September, 26 & September, 25 \\
$20 \mathrm{~cm} / 3$ cuts & September, 16 & September, 19 & September, 26 & September, 28 \\
$30 \mathrm{~cm} / 1$ cut & September, 9 & September, 16 & September, 22 & September, 20 \\
$30 \mathrm{~cm} / 2$ cuts & September, 24 & September, 22 & October, 2 & October, 15 \\
$30 \mathrm{~cm} / 3$ cuts & September, 30 & September, 29 & October, 9 & October, 12 \\
\hline
\end{tabular}

Productivity and bromatological composition of forage

Regardless the canopy height, for two cultivars and in two crop season, accumulated forage productivity (FP) increased with increase in cuts number. For FP obtained, in same cut, at different canopy heights, a higher value was obtained in plants managed in $30 \mathrm{~cm}$ (Table 3 ). Similar behavior was obtained in other studies such as Tonetto et al. (2011) who observed that in ryegrass, the increase in cuts number resulted in increased dry matter production. Ramella et al.
(2015) found that in dual-purpose wheat BRS cultivar Tarumã, FP was higher in two cuts compared to single cut. Hastenpflug et al. (2011a) working with four dual-purpose wheat cultivars, managed in two cuts, obtained that in second cut, the FP practically doubled. Tian et al. (2012) in an experiment conducted with dual-purpose wheat, managed in three cuts according to plant phenological stage (before and after GS 30), verified increases in FP, because the crop was in vegetative growth stage, which can also be verified in this work.

Table 3. Accumulated forage productivity (DM) and tiller population density of dual-purpose wheat BRS Umbu and BRS Tarumã cultivars, submitted to two canopy heights and cuts number, in two crop seasons.

\begin{tabular}{|c|c|c|c|c|c|c|}
\hline \multirow[b]{4}{*}{ Cuts number } & \multicolumn{6}{|c|}{ Canopy heights $(\mathrm{cm})$} \\
\hline & 20 & 30 & & 20 & 30 & \\
\hline & \multicolumn{6}{|c|}{----------- Accumulated forage productivity BRS Umbu $\left(\mathrm{kg} \mathrm{DM} \mathrm{ha}^{-1}\right)$------------- } \\
\hline & \multicolumn{2}{|c|}{2013} & Mean & \multicolumn{2}{|c|}{2014} & \multirow{2}{*}{$\frac{\text { Mean }}{283.3}$} \\
\hline 1 & $192.2 \mathrm{c} \mathrm{B}$ & 392.9 c A & 292.5 & $175.0 \mathrm{c} \mathrm{B}$ & $391.6 \mathrm{c} \mathrm{A}$ & \\
\hline 2 & $565.4 \mathrm{~b} \mathrm{~B}$ & $1217.3 \mathrm{~b} \mathrm{~A}$ & 891.3 & 529.1 b B & $1004.1 \mathrm{~b} \mathrm{~A}$ & 766.6 \\
\hline 3 & 1044.5 a B & 1866.1 a A & 1455.3 & 825.0 a B & $1320.0 \mathrm{a} \mathrm{A}$ & 1072.5 \\
\hline Mean & 600.8 & 1158.7 & 879.7 & 509.7 & 905.2 & 707.5 \\
\hline CV $(\%)$ & & 14.2 & & & 15.1 & \\
\hline \multicolumn{7}{|c|}{ Source of variation } \\
\hline $\mathrm{H}$ & & $<0.001$ & & \multicolumn{3}{|c|}{$<0.001$} \\
\hline $\mathrm{C}$ & & $<0.001$ & & \multicolumn{3}{|c|}{$<0.001$} \\
\hline $\mathrm{Hx} \mathrm{C}$ & & $<0.001$ & & & 0.035 & \\
\hline \multirow[b]{2}{*}{ Cuts number } & \multicolumn{6}{|c|}{--------- Accumulated forage productivity BRS Tarumã $\left(\mathrm{kg} \mathrm{DM} \mathrm{ha}^{-1}\right)$} \\
\hline & \multicolumn{2}{|c|}{2014} & Mean & \multicolumn{2}{|c|}{2015} & Mean \\
\hline 1 & 191.6 c B & 687.5 c A & 439.5 & $491.7 \mathrm{~b} \mathrm{~B}$ & $1041.7 \mathrm{~b} \mathrm{~A}$ & 766.7 \\
\hline 2 & $758.3 \mathrm{~b} \mathrm{~B}$ & $1162.5 \mathrm{~b} \mathrm{~A}$ & 960.4 & 1333.3 a A & $1220.8 \mathrm{~b} \mathrm{~A}$ & 1277.1 \\
\hline 3 & 1041.6 a B & 1895.8 a A & 1468.7 & 1637.5 a B & 2079.2 a A & 1858.3 \\
\hline Mean & 663.8 & 1248.6 & 956.2 & 1154.1 & 1447.2 & 1300.7 \\
\hline CV $(\%)$ & & 9.4 & & & 18.3 & \\
\hline \multicolumn{7}{|c|}{ Source of variation } \\
\hline $\mathrm{H}$ & & $<0.001$ & & & 0.008 & \\
\hline $\mathrm{C}$ & & $<0.001$ & & & $<0.001$ & \\
\hline $\mathrm{H} \times \mathrm{C}$ & & $<0.001$ & & & 0.030 & \\
\hline
\end{tabular}




\begin{tabular}{|c|c|c|c|c|c|c|}
\hline \multirow{2}{*}{$\begin{array}{c}\text { Cuts number } \\
0\end{array}$} & \multicolumn{2}{|c|}{2013} & \multirow{2}{*}{$\frac{\text { Mean }}{851}$} & \multicolumn{2}{|c|}{2014} & \multirow{2}{*}{$\begin{array}{c}\text { Mean } \\
931\end{array}$} \\
\hline & 841 a A & 860 a A & & 903 a $\mathrm{A}$ & 960 a A & \\
\hline 1 & 866 a A & 827 a A & 847 & 964 a A & 905 a A & 935 \\
\hline 2 & 860 a A & 802 a B & 831 & $879 \mathrm{ab} A$ & $790 \mathrm{~b} \mathrm{~B}$ & 835 \\
\hline 3 & $785 \mathrm{~b} \mathrm{~A}$ & $719 \mathrm{~b} \mathrm{~B}$ & 752 & 798 b A & $701 \mathrm{c} \mathrm{B}$ & 749 \\
\hline Mean & 838 & 802 & 820 & 886 & 839 & 862 \\
\hline $\mathrm{CV}(\%)$ & & 3.4 & & & 4.7 & \\
\hline \multicolumn{7}{|c|}{ Source of variation } \\
\hline $\mathrm{H}$ & & 0.001 & & & 0.003 & \\
\hline $\mathrm{C}$ & & $<0.001$ & & & $<0.001$ & \\
\hline $\mathrm{HxC}$ & & 0.029 & & & 0.003 & \\
\hline & \multicolumn{6}{|c|}{ 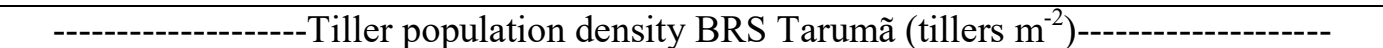 } \\
\hline Cuts number & \multicolumn{2}{|c|}{2014} & Mean & \multicolumn{2}{|c|}{2015} & Mean \\
\hline 0 & $1136 \mathrm{~b} \mathrm{~A}$ & 1155 a $\mathrm{A}$ & 1145 & 2194 b A & 2172 a A & 2183 \\
\hline 1 & $1175 \mathrm{~b} \mathrm{~A}$ & 1254 a A & 1214 & $2229 \mathrm{~b} \mathrm{~A}$ & 2177 a A & 2203 \\
\hline 2 & 1428 a A & 1268 a B & 1348 & 2511 a A & 2218 a B & 2364 \\
\hline 3 & 1347 a A & $950 \mathrm{~b} \mathrm{~B}$ & 1148 & 2482 a A & $1876 \mathrm{~b} \mathrm{~B}$ & 2180 \\
\hline Mean & 1272 & 1156 & 1214 & 2354 & 2111 & 2232 \\
\hline CV $(\%)$ & & 5.8 & & & 2.4 & \\
\hline \multicolumn{7}{|c|}{ Source of variation } \\
\hline $\mathrm{H}$ & & $<0.001$ & & & $<0.001$ & \\
\hline $\mathrm{C}$ & & $<0.001$ & & & $<0.001$ & \\
\hline $\mathrm{H} \times \mathrm{C}$ & & $<0.001$ & & & $<0.001$ & \\
\hline
\end{tabular}

CV: Coefficient of variation; H: canopy height; C: cuts number; *Means followed by the same letter, lowercase within column and uppercase within row, do not differ by Tukey test at $5 \%$ probability.

Different from results described, possibly due to the occurrence of higher temperatures in vegetative growth period, in BRS Tarumã cultivar 2015 crop season, the PF of plants managed at $20 \mathrm{~cm}$ in second cut was substantially similar to third cutting. Following the same trend, due to climatic conditions, $\mathrm{PF}$ in second cut between two canopy heights were similar (Table 3).

Since tiller is the grasses basic vegetative unit (HODGSON, 1990), TPD exploration, which is a pasture structural characteristic, helps define management strategies for forage plants (ARAÚJO et al., 2015). According to Bortolini et al. (2004), increase in DM associated at multiple cuts can be explained by high regrowth capacity and new tillers formation. Corroborating with these authors, it was observed that FP increase may be related to a general TPD stability, for BRS Umbu cultivar. For BRS Tarumã cultivar, it was observed that TPD in plants managed at $20 \mathrm{~cm}$ increased from second cut, where the late cycle of this cultivar is related to results obtained (Table 3 ).

However, TPD decreased prominently in third cut, allied to $30 \mathrm{~cm}$ (Table 3 ). This reduction may be associated to tillering stage closure (beyond GS 30), a possible tiller death associated with apical meristems removal (plants do not emit new tillers sufficiently to compensate the population) and due to leaf area reductions, that causes stress and less tiller regrowth (TIAN et al., 2012; NAVEED et al., 2014).

In this way, it can be observed that FP is related to TPD, especially in plants managed at 30 $\mathrm{cm}$, since FP increase with cuts number increase can be maintained due to a compensatory mechanism of tiller size/ density and self-thinning promoted by light limitation (YODA et al., 1963; MATTHEW et al., 1995). Thus, it was observed that plants managed at $20 \mathrm{~cm}$ showed more tillers than $30 \mathrm{~cm}$, but a FP was higher in this last height, because tillers were in smaller number, but which a higher density (Table 3).

Wheat forage bromatological composition is high, rich in protein and low fiber, and plants nutritional levels vary throughout developmental stages, decreasing the $\mathrm{CP}$ and raising the fibers percentage (Naveed et al., 2015). Based on this assertion, it can be observed that in this work, forage bromatological composition levels of two wheat cultivars behaved according to plant phenology in cut moments (Table 4). 
Table 4. Crude protein, neutral detergent fiber and acid detergent fiber of dual-purpose wheat BRS Umbu and BRS Tarumã cultivars, submitted to two canopy heights and cuts number, in two crop seasons.

\begin{tabular}{|c|c|c|c|c|c|c|}
\hline \multirow{4}{*}{ Cuts number } & \multicolumn{6}{|c|}{ Canopy heights $(\mathrm{cm})$} \\
\hline & 20 & 30 & & 20 & 30 & \\
\hline & \multicolumn{6}{|c|}{ 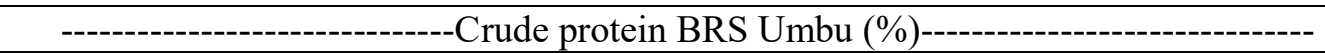 } \\
\hline & \multicolumn{2}{|c|}{2013} & Mean & \multicolumn{2}{|c|}{2014} & \multirow{2}{*}{$\frac{\text { Mear }}{28.6}$} \\
\hline 1 & $29.0 \mathrm{a} \mathrm{A}^{\dagger}$ & 23.4 a B & 26.2 & 30.0 a $\mathrm{A}$ & 27.2 a B & \\
\hline 2 & 28.5 a A & $20.5 \mathrm{~b} \mathrm{~B}$ & 24.5 & 29.9 a $\mathrm{A}$ & $25.5 \mathrm{~b} \mathrm{~B}$ & 27.7 \\
\hline 3 & $26.0 \mathrm{~b} \mathrm{~A}$ & $18.5 \mathrm{c} \mathrm{B}$ & 22.2 & 27.9 a $\mathrm{A}$ & $25.4 \mathrm{~b} \mathrm{~B}$ & 26.7 \\
\hline Mean & 27.8 & 20.8 & 24.3 & 29.2 & 26.1 & 27.6 \\
\hline CV (\%) & & 3.0 & & & 2.8 & \\
\hline \multicolumn{7}{|c|}{ Source of variation } \\
\hline $\mathrm{H}$ & & $<0.001$ & & & $<0.001$ & \\
\hline C & & $<0.001$ & & & 0.007 & \\
\hline $\mathrm{H} \times \mathrm{C}$ & & 0.009 & & & 0.043 & \\
\hline
\end{tabular}

\begin{tabular}{|c|c|c|c|c|c|c|}
\hline \multirow[b]{2}{*}{ Cuts number } & \multicolumn{6}{|c|}{ 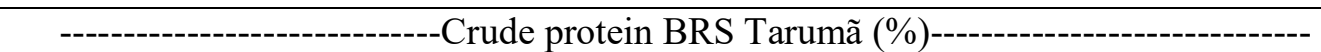 } \\
\hline & \multicolumn{2}{|c|}{2014} & \multirow{2}{*}{$\begin{array}{c}\text { Mean } \\
23.7\end{array}$} & \multicolumn{2}{|c|}{2015} & \multirow{2}{*}{$\begin{array}{c}\text { Mean } \\
23.0\end{array}$} \\
\hline 1 & $29.4 \mathrm{~b} \mathrm{~A}$ & $18.1 \mathrm{~b} \mathrm{~B}$ & & $26.0 \mathrm{~b} \mathrm{~A}$ & $20.1 \mathrm{~b} \mathrm{~B}$ & \\
\hline 2 & $28.6 \mathrm{~b} \mathrm{~A}$ & 26.3 a B & 27.4 & $26.6 \mathrm{~b} \mathrm{~A}$ & 24.9 a B & 25.7 \\
\hline 3 & $32.2 \mathrm{a} \mathrm{A}$ & 26.2 a B & 29.2 & 28.9 a $\mathrm{A}$ & 25.8 a B & 27.4 \\
\hline Mean & 30.0 & 23.5 & 26.8 & 27.2 & 23.6 & 25.4 \\
\hline CV $(\%)$ & & 3.7 & & & 4.8 & \\
\hline \multicolumn{7}{|c|}{ Source of variation } \\
\hline $\mathrm{H}$ & & $<0.001$ & & & $<0.001$ & \\
\hline $\mathrm{C}$ & & $<0.001$ & & & $<0.001$ & \\
\hline $\mathrm{H} \times \mathrm{C}$ & & $<0.001$ & & & $<0.001$ & \\
\hline
\end{tabular}

\begin{tabular}{|c|c|c|c|c|c|c|}
\hline \multirow[b]{2}{*}{ Cuts number } & \multicolumn{6}{|c|}{ 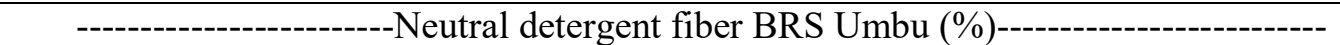 } \\
\hline & \multicolumn{2}{|c|}{2013} & \multirow{2}{*}{$\begin{array}{c}\text { Mean } \\
40.4\end{array}$} & \multicolumn{2}{|c|}{2014} & \multirow{2}{*}{$\begin{array}{c}\text { Mean } \\
36.6\end{array}$} \\
\hline 1 & $39.6 \mathrm{~b} \mathrm{~A}$ & $41.1 \mathrm{c} \mathrm{A}$ & & 34.3 a B & $38.8 \mathrm{c} \mathrm{A}$ & \\
\hline 2 & 43.6 a B & $49.3 \mathrm{~b} \mathrm{~A}$ & 46.5 & 34.9 a B & $46.1 \mathrm{~b} \mathrm{~A}$ & 40.5 \\
\hline 3 & $41.0 \mathrm{ab} B$ & 58.3 a $\mathrm{A}$ & 49.6 & 35.6 a B & 50.3 a $\mathrm{A}$ & 42.9 \\
\hline Mean & 41.4 & 49.6 & 45.5 & 34.9 & 45.1 & 40.0 \\
\hline $\mathrm{CV}(\%)$ & & 4.7 & & & 3.5 & \\
\hline \multicolumn{7}{|c|}{ Source of variation } \\
\hline $\mathrm{H}$ & & $<0.001$ & & & $<0.001$ & \\
\hline C & & $<0.001$ & & & $<0.001$ & \\
\hline $\mathrm{H} \times \mathrm{C}$ & & $<0.001$ & & & $<0.001$ & \\
\hline
\end{tabular}

\begin{tabular}{|c|c|c|c|c|c|c|}
\hline \multirow[b]{2}{*}{ Cuts number } & \multicolumn{6}{|c|}{ 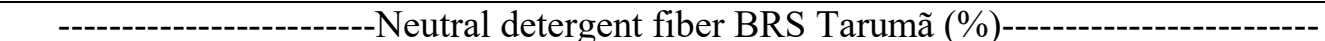 } \\
\hline & \multicolumn{2}{|c|}{2014} & Mean & \multicolumn{2}{|c|}{2015} & Mean \\
\hline 1 & $33.1 \mathrm{~b} \mathrm{~B}$ & $45.6 \mathrm{~b} \mathrm{~A}$ & 39.3 & 48.9 a A & $50.3 \mathrm{c} \mathrm{A}$ & 49.6 \\
\hline 2 & 44.2 a B & $47.0 \mathrm{~b} \mathrm{~A}$ & 45.6 & 50.2 a B & $54.4 \mathrm{~b} \mathrm{~A}$ & 52.3 \\
\hline 3 & 44.4 a B & $53.2 \mathrm{a} \mathrm{A}$ & 48.8 & 48.9 a B & 58.9 a $\mathrm{A}$ & 53.9 \\
\hline Mean & 40.5 & 48.6 & 44.6 & 49.4 & 54.5 & 51.9 \\
\hline CV $(\%)$ & & 3.4 & & & 1.9 & \\
\hline \multicolumn{7}{|c|}{ Source of variation } \\
\hline $\mathrm{H}$ & & $<0.001$ & & & $<0.001$ & \\
\hline $\mathrm{C}$ & & $<0.001$ & & & $<0.001$ & \\
\hline $\mathrm{H} \times \mathrm{C}$ & & $<0.001$ & & & $<0.01$ & \\
\hline
\end{tabular}

\begin{tabular}{|c|c|c|c|c|c|c|}
\hline \multirow[b]{2}{*}{ Cuts number } & \multicolumn{6}{|c|}{ - } \\
\hline & \multicolumn{2}{|c|}{2013} & Mean & \multicolumn{2}{|c|}{2014} & Mean \\
\hline 1 & $17.5 \mathrm{c} \mathrm{B}$ & $20.7 \mathrm{c} \mathrm{A}$ & 19.1 & $15.7 \mathrm{~b} \mathrm{~B}$ & $18.8 \mathrm{c} \mathrm{A}$ & 17.4 \\
\hline 2 & $20.7 \mathrm{~b} \mathrm{~B}$ & $24.0 \mathrm{~b} \mathrm{~A}$ & 23.5 & 18.0 a B & $22.8 \mathrm{~b} \mathrm{~A}$ & 19.2 \\
\hline 3 & $23.1 \mathrm{a} \mathrm{B}$ & 28.8 a $\mathrm{A}$ & 24.7 & 18.9 a B & 25.9 a $\mathrm{A}$ & 22.4 \\
\hline Mean & 20.4 & 24.5 & 22.5 & 17.5 & 21.9 & 19.7 \\
\hline
\end{tabular}




\begin{tabular}{|c|c|c|c|c|c|c|}
\hline CV (\%) & & 4.2 & & & 3.5 & \\
\hline Source of var & & & & & & \\
\hline $\mathrm{H}$ & & $<0.001$ & & & $<0.001$ & \\
\hline $\mathrm{C}$ & & $<0.001$ & & & $<0.001$ & \\
\hline $\mathrm{H} \times \mathrm{C}$ & & $<0.001$ & & & $<0.001$ & \\
\hline & --------. & ------------ & tergent & RS Tarum & )-----------. & - \\
\hline Cuts number & & & Mean & & & Mean \\
\hline 1 & $15.4 \mathrm{c} \mathrm{B}$ & $20.0 \mathrm{c} \mathrm{A}$ & 17.7 & 19.5 c B & $20.8 \mathrm{c} \mathrm{A}$ & 20.2 \\
\hline 2 & $18.6 \mathrm{~b} \mathrm{~B}$ & $21.7 \mathrm{~b} \mathrm{~A}$ & 20.1 & $21.6 \mathrm{~b} \mathrm{~B}$ & $24.3 \mathrm{~b} \mathrm{~A}$ & 22.9 \\
\hline 3 & 20.9 a B & 24.6 a A & 22.7 & $23.6 \mathrm{a} \mathrm{B}$ & $25.9 \mathrm{a} \mathrm{A}$ & 24.7 \\
\hline Mean & 18.3 & 22.1 & 20.2 & 21.6 & 23.7 & 22.6 \\
\hline CV (\%) & & 2.3 & & & 1.8 & \\
\hline Source of var & & & & & & \\
\hline $\mathrm{H}$ & & $<0.001$ & & & $<0.001$ & \\
\hline $\mathrm{C}$ & & $<0.001$ & & & $<0.001$ & \\
\hline $\mathrm{H} \times \mathrm{C}$ & & 0.021 & & & 0.011 & \\
\hline
\end{tabular}

CV: Coefficient of variation; H: canopy height; C: cuts number; *Means followed by the same letter, lowercase within column and uppercase within row, do not differ by Tukey test at $5 \%$ probability.

Crude protein levels were elevated according to NRC requirements (2001), which address 18 to $20 \%$ of this fraction to animal nutrition. Nitrogen content excess may have been derived from replacement fertilization, ends up being excreted in urine and is not used in diet (VAN SOEST et al., 1991). Thus, reductions in CP levels found in forage from BRS Umbu cultivar, in third cut to $20 \mathrm{~cm}$ and second cut to $30 \mathrm{~cm}$, may be nutritionally beneficial (Table 4). In contrast to present work, Tian et al. (2012) found that CP content was similar across cuts before GS 30 and after this stage there was a decrease of the same. Crude protein content increase with cuts number increase observed in BRS Tarumã cultivar (Table 4) may be related to the findings of MacKown and Carver (2005), that late maturing cultivars have a higher plant nitrogen concentration. Content above
$60 \%$ NDF and $40 \%$ ADF are limiting to consumption and forage digestibility, respectively (VAN SOEST, 1965). It can be observed, as Meinerz et al. (2011), that for both cultivars, NDF and ADF levels increased with cuts number increase, following the plant development, but were below the limits cited above (Table 4).

\section{Grain yield and yield components}

Concerning grain yield, it was observed that for BRS Umbu cultivar in both crop seasons and BRS Tarumã 2014 crop season, the GY did not present a significant difference between the cuts numbers in plants managed at $20 \mathrm{~cm}$. For BRS Umbu and BRS Tarumã 2014 crop season, the GY decreased in plants managed at $30 \mathrm{~cm}$ with three cuts (Table 5).

Table 5. Grain yield and plant height of dual-purpose wheat BRS Umbu and BRS Tarumã cultivars, submitted to two canopy heights and cuts number, in two crop seasons.

\begin{tabular}{|c|c|c|c|c|c|c|}
\hline \multirow[b]{4}{*}{ Cuts number } & \multicolumn{6}{|c|}{ Canopy heights $(\mathrm{cm})$} \\
\hline & 20 & 30 & & 20 & 30 & \\
\hline & \multicolumn{6}{|c|}{ 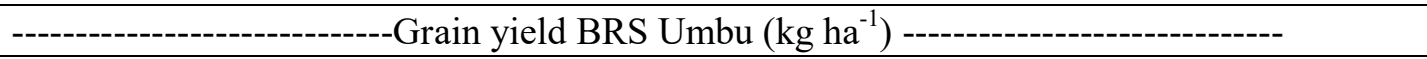 } \\
\hline & \multicolumn{2}{|c|}{2013} & Mean & \multicolumn{2}{|c|}{2014} & Mean \\
\hline 0 & $3164.1 \mathrm{a} \mathrm{A}^{\dagger}$ & 2579.9 a A & 2872.0 & 4874.6 a A & $4664.5 \mathrm{a} \mathrm{A}$ & 4769.6 \\
\hline 1 & 2974.7 a A & 2731.9 a A & 2853.3 & 4327.0 a A & 4439.1 a $\mathrm{A}$ & 4383.0 \\
\hline 2 & 2665.7 a A & $2438.1 \mathrm{a} \mathrm{A}$ & 2551.9 & 4661.4 a A & 4425.3 a A & 4543.3 \\
\hline 3 & $3041.1 \mathrm{a} \mathrm{A}$ & 1019.3 b B & 2030.2 & 4525.5 a A & 3244.3 b B & 3884.9 \\
\hline Mean & 2961.4 & 2192.3 & 2576.8 & 4597.1 & 4193.30 & 4395.2 \\
\hline CV (\%) & & 20.3 & & & 8.9 & \\
\hline \multicolumn{7}{|c|}{ Source of variation } \\
\hline $\mathrm{H}$ & & 0.004 & & & 0.008 & \\
\hline $\mathrm{C}$ & & 0.013 & & & 0.001 & \\
\hline
\end{tabular}




\begin{tabular}{|c|c|c|c|c|c|c|}
\hline \multirow[t]{2}{*}{$\mathrm{Hx} \mathrm{C}$} & \multicolumn{3}{|c|}{0.007} & \multicolumn{3}{|c|}{0.010} \\
\hline & \multicolumn{6}{|c|}{ 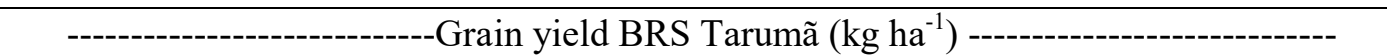 } \\
\hline Cuts number & \multicolumn{2}{|c|}{2014} & Mean & \multicolumn{2}{|c|}{2015} & Mean \\
\hline 0 & 4392.3 a A & $4054.4 \mathrm{ab} \mathrm{A}$ & 4223.3 & $371.0 \mathrm{a} \mathrm{A}$ & $467.0 \mathrm{ab} \mathrm{A}$ & 419.0 \\
\hline 1 & $4500.0 \mathrm{a} \mathrm{A}$ & 4367.5 a A & 4433.7 & 450.7 a A & 499.0 a A & 474.9 \\
\hline 2 & 4167.2 a A & $3628.6 \mathrm{bc} \mathrm{B}$ & 3897.9 & $440.2 \mathrm{a} \mathrm{A}$ & $373.4 \mathrm{~b} \mathrm{~A}$ & 406.8 \\
\hline 3 & 4167.7 a A & 2991.5 c B & 3579.6 & $280.7 \mathrm{~b} \mathrm{~B}$ & $363.8 \mathrm{~b} \mathrm{~A}$ & 322.2 \\
\hline Mean & 4306.8 & 3760.5 & 4033.63 & 385.6 & 425.8 & 405.7 \\
\hline CV $(\%)$ & & 7.5 & & & 12.2 & \\
\hline \multicolumn{7}{|c|}{ Source of variation } \\
\hline $\mathrm{H}$ & & $<0.001$ & & & 0.024 & \\
\hline $\mathrm{C}$ & & $<0.001$ & & & $<0.001$ & \\
\hline \multirow[t]{2}{*}{$\mathrm{H} \times \mathrm{C}$} & & 0.013 & & & 0.031 & \\
\hline & \multicolumn{6}{|c|}{ - } \\
\hline Cuts number & \multicolumn{2}{|c|}{2013} & Mean & \multicolumn{2}{|c|}{2014} & Mean \\
\hline 0 & 88.0 a $\mathrm{A}$ & $87.5 \mathrm{a} \mathrm{A}$ & 87.7 & $85.5 \mathrm{ab} A$ & 86.0 a $\mathrm{A}$ & 85.7 \\
\hline 1 & $83.1 \mathrm{ab} A$ & 81.8 a A & 82.4 & 88.6 a A & 85.4 a A & 87.0 \\
\hline 2 & $80.0 \mathrm{~b} \mathrm{~A}$ & $70.7 \mathrm{~b} \mathrm{~B}$ & 75.4 & $85.8 \mathrm{ab} \mathrm{A}$ & $79.6 \mathrm{ab} B$ & 82.7 \\
\hline 3 & $74.5 \mathrm{c} \mathrm{A}$ & $65.3 \mathrm{~b} \mathrm{~B}$ & 69.9 & $83.6 \mathrm{~b} \mathrm{~A}$ & $75.4 \mathrm{~b} \mathrm{~B}$ & 79.5 \\
\hline Mean & 81.45 & 76.3 & 78.8 & 85.9 & 81.6 & 83.7 \\
\hline CV (\%) & & 3.6 & & & 2.9 & \\
\hline \multicolumn{7}{|c|}{ Source of variation } \\
\hline $\mathrm{H}$ & & $<0.001$ & & & $<0.001$ & \\
\hline $\mathrm{C}$ & & $<0.001$ & & & $<0.001$ & \\
\hline $\mathrm{H} \times \mathrm{C}$ & & 0.006 & & & 0.012 & \\
\hline
\end{tabular}

\begin{tabular}{|c|c|c|c|c|c|c|}
\hline \multirow{3}{*}{$\frac{\text { Cuts number }}{0}$} & \multicolumn{6}{|c|}{ 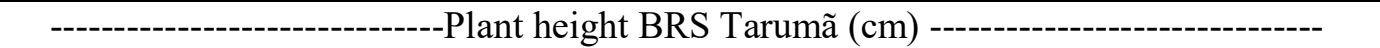 } \\
\hline & \multicolumn{2}{|c|}{2014} & \multirow{2}{*}{$\begin{array}{c}\text { Mean } \\
75.8\end{array}$} & \multicolumn{2}{|c|}{2015} & \multirow{2}{*}{$\begin{array}{l}\text { Mean } \\
59.6\end{array}$} \\
\hline & 76.6 a $\mathrm{A}$ & 75.0 a A & & $59.3 \mathrm{a} \mathrm{A}$ & $59.2 \mathrm{a} \mathrm{A}$ & \\
\hline 1 & 77.0 a $\mathrm{A}$ & 72.8 a B & 74.9 & $56.3 \mathrm{ab} \mathrm{A}$ & $55.0 \mathrm{ab} \mathrm{A}$ & 55.6 \\
\hline 2 & $73.8 \mathrm{~b} \mathrm{~A}$ & $64.8 \mathrm{~b} \mathrm{~B}$ & 69.3 & $52.3 \mathrm{~b} \mathrm{~A}$ & $50.4 \mathrm{bc} \mathrm{A}$ & 51.3 \\
\hline 3 & $71.8 \mathrm{~b} \mathrm{~A}$ & $54.3 \mathrm{c} \mathrm{B}$ & 63.0 & $53.5 \mathrm{~b} \mathrm{~A}$ & 44.2 c B & 48.8 \\
\hline Mean & 74.8 & 66.7 & 70.7 & 55.5 & 52.2 & 53.8 \\
\hline CV (\%) & & 2.4 & & & 4.7 & \\
\hline \multicolumn{7}{|c|}{ Source of variation } \\
\hline $\mathrm{H}$ & & $<0.001$ & & & 0.001 & \\
\hline $\mathrm{C}$ & & $<0.0001$ & & & $<0.001$ & \\
\hline $\mathrm{H} \times \mathrm{C}$ & & $<0.001$ & & & 0.008 & \\
\hline
\end{tabular}

CV: Coefficient of variation; H: canopy height; C: cuts number; *Means followed by the same letter, lowercase within column and uppercase within row, do not differ by Tukey test at $5 \%$ probability.

It is possible to emphasize that plant nitrogen nutrition contributed to this results in GY, because the shoots that acted as source organs were satisfactory to supply the grains production, sink organs. With the cuts made throughout the plant cycle, apical meristems decapitation and height of plants decrease occurred, with consequent reduction in GY, mainly employing $30 \mathrm{~cm}$ management. Thus, the results showed that the cuts influenced the height of plants for both cultivars, from the second cut, contributing to GY reduce in the third cut (Table 5).

For BRS Tarumã cultivar in 2015 crop season, in plants managed at $20 \mathrm{~cm}$ was found a significant difference in GY, as a function of cuts $(p<0.05)$. According to 2015 crop season meteorological data, shown in Figure 1, the precipitation amounts at time of anthesis and the freezing temperatures occurrence in this culture critical period, may have affected the GY (Table 
5). Therefore, it can be observed that even in years with climatic adversities that contribute to GY reduction, it is possible to produce forage, so that it does not affect the production system as a whole.

Our results confirm that factors such as defoliation proportion influence on grain production. Conditions related to apical meristems removal by cutting or grazing, remaining leaf area proportion and the consequent reduction radiation interception and photoassimilates distribution, cuts or grazing beyond GS 30, delayed the maturation and shortened the grain filling period, resulting in grain yield decrease (BELL et al., 2015; HARRISON et al., 2011; HARRISON et al., 2014; SEYMOUR et al., 2015). Similar to this study, Hastenpflug et al. (2011b), Carletto et al. (2015), and Ramella et al. (2015) made two cuts in wheat and observed that the grain yield was reduced due to cuts. Tian et al. (2012) found that wheat defoliated after GS 30 resulted in reduction of more than $60 \%$ in grain yield, mainly due to meristems removal, subsequent tillers death and reduction of plant capacity to translocate resources for grain production and filling.

In this work, TGW was reduced from second cut for both cultivars and crop seasons, regardless of canopy height used (Table 6). In addition, the lower TGW of BRS Tarumã cultivar was due to this one having smaller grains and consequently lower mass (SANTOS et al., 2015). This decrease in TGW can be related to stress caused by defoliation, resulting in reduced grains photoassimilates distribution (NAVEED et al., 2014) and also by reduced grain filling period as a function of delayed anthesis dates compared to control (Table 2). Carletto et al. (2015) reported that cuts management influenced TGW and observed that in BRS Umbu cultivar this characteristic was significantly lower as a function of second cut performed. Islam et al. (2015) also found that TGW was reduced due to defoliation in dual-purpose wheat.

Table 6. Thousand grain weight, number of spikelets per spike and number of grains per spike of dual-purpose wheat BRS Umbu and BRS Tarumã cultivars, submitted to cuts number, in two crop seasons.

\begin{tabular}{|c|c|c|c|c|}
\hline \multirow[b]{2}{*}{ Cuts number } & 2013 & 2014 & 2014 & 2015 \\
\hline & \multicolumn{2}{|c|}{ Weight of one thousand grain BRS --- } & \multicolumn{2}{|c|}{$\begin{array}{l}\text { Weight of one thousand grain BRS } \\
\text { - }\end{array}$} \\
\hline c & $31.3 \mathrm{ab}^{\dagger}$ & $31.6 \mathrm{a}$ & $27.5 \mathrm{a}$ & $20.4 \mathrm{a}$ \\
\hline 1 & $32.5 \mathrm{a}$ & $30.4 \mathrm{a}$ & $27.2 \mathrm{a}$ & $20.0 \mathrm{a}$ \\
\hline 2 & $28.7 \mathrm{~b}$ & $27.1 \mathrm{~b}$ & $25.7 \mathrm{~b}$ & $18.4 \mathrm{~b}$ \\
\hline 3 & $28.9 \mathrm{~b}$ & $27.8 \mathrm{~b}$ & $24.9 \mathrm{~b}$ & $18.5 \mathrm{~b}$ \\
\hline Mean & 30.4 & 29.2 & 26.4 & 19.3 \\
\hline CV $(\%)$ & 7.2 & 4.8 & 3.2 & 3.3 \\
\hline \multicolumn{5}{|c|}{ Source of variation } \\
\hline $\mathrm{H}$ & 0.116 & 0.765 & 0.101 & 0.372 \\
\hline $\mathrm{C}$ & 0.005 & $<0.001$ & $<0.001$ & $<0.001$ \\
\hline $\mathrm{Hx} \mathrm{C}$ & 0.119 & 0.179 & 0.168 & 0.207 \\
\hline Cuts number & \multicolumn{2}{|c|}{$\begin{array}{l}\text { Number of spikelets per spike } \\
-\end{array}$} & \multicolumn{2}{|c|}{$\begin{array}{l}\text { Number of spikelets per spike } \\
\text {-------BRS Tarumã-------- }\end{array}$} \\
\hline c & $12.1 \mathrm{a}$ & $13.8 \mathrm{a}$ & $11.8 \mathrm{ab}$ & $14.1 \mathrm{a}$ \\
\hline 1 & $11.6 \mathrm{ab}$ & $13.3 \mathrm{a}$ & $12.5 \mathrm{a}$ & $13.3 \mathrm{ab}$ \\
\hline 2 & $11.5 \mathrm{ab}$ & $13.0 \mathrm{a}$ & $11.0 \mathrm{ab}$ & $12.6 \mathrm{~b}$ \\
\hline 3 & $10.8 \mathrm{~b}$ & $11.0 \mathrm{~b}$ & $10.3 \mathrm{~b}$ & $11.5 \mathrm{c}$ \\
\hline Mean & 11.5 & 12.8 & 11.4 & 12.9 \\
\hline CV (\%) & 6.0 & 8.9 & 10.6 & 5.9 \\
\hline \multicolumn{5}{|c|}{ Source of variation } \\
\hline $\mathrm{H}$ & 0.217 & 0.079 & 0.260 & 0.497 \\
\hline $\mathrm{C}$ & 0.015 & $<0.001$ & 0.0114 & $<0.001$ \\
\hline $\mathrm{H} \times \mathrm{C}$ & 0.126 & 0.903 & 0.387 & 0.079 \\
\hline Cuts number & Number & er spike & $\begin{array}{l}\text { Number } \\
-----B R S\end{array}$ & $\begin{array}{l}\text { per spike } \\
\text { p 2014----- }\end{array}$ \\
\hline
\end{tabular}




\begin{tabular}{|c|c|c|c|}
\hline & $22.7 \mathrm{a}$ & $24.8 \mathrm{a}$ & $18.2 \mathrm{ab}$ \\
\hline & $21.2 \mathrm{a}$ & $22.4 \mathrm{ab}$ & $18.4 \mathrm{a}$ \\
\hline & $18.0 \mathrm{ab}$ & $19.6 \mathrm{bc}$ & $16.5 \mathrm{ab}$ \\
\hline & $15.8 \mathrm{~b}$ & $15.5 \mathrm{c}$ & $14.2 \mathrm{~b}$ \\
\hline Mean & 19.4 & 20.5 & 16.8 \\
\hline CV (\%) & 18.8 & 15.5 & 17.5 \\
\hline Source of & & & \\
\hline $\mathrm{H}$ & 0.761 & 0.115 & 0.532 \\
\hline C & 0.004 & $<0.001$ & 0.035 \\
\hline $\mathrm{H} \times \mathrm{C}$ & 0.058 & 0.098 & 0.978 \\
\hline
\end{tabular}

CV: Coefficient of variation; H: canopy height; C: cuts number; *Means followed by the same letter, lowercase within column and uppercase within row, do not differ by Tukey test at $5 \%$ probability.

According to Hendrickson et al. (2005), when the apical meristem is removed, the secondary tiller appearance is induced and plants produce smaller spikes with few grains. The yield components NSS and NGS were reduced in third cut in both cultivars and crop seasons, regardless of canopy height employed (Table 6), except for NGS of BRS Tarumã cultivar 2015 crop season, where no significant differences were found $(p<0.05)$. For these components, Hastenpflug et al. (2011b) found that NSS decreased with cuts. Under two cuts management Ramella et al. (2015) too found that NSS and NGS decreased. Virgona et al. (2006) reported a decrease of $50-60 \%$ in NGS due to prolonged grazing periods and Tian et al. (2012) reported that cuts after GS 30 resulted in $55 \%$ NGS reduction compared to non-defoliated plants.

\section{CONCLUSIONS}

Results show that canopy heights and cuts number led to differences in productive characteristics of wheat BRS Umbu and
BRS Tarumã cultivars in dual- purpose system. The trend of the results due treatments were similar for the two cultivars of different growth habits, except for the trait tillering. Therefore, management strategies can be selected according to the situation and farmer intended purpose in profiting more with either the forage harvest or grain. These two wheat cultivars can be employed for dual-purpose with forage production plus grain and may be an approach suited to southern Brazil cropping and grazing systems. In dual-purpose wheat managed at $20 \mathrm{~cm}$, it is possible to obtain forage for animals, prioritizing grain yield. At $30 \mathrm{~cm}$ forage yield was prioritized and grain yield was penalized, as a result of the third cut for both cultivars.

\section{ACKNOWLEDGMENTS}

The authors would like to thank Brazilian's fellowships: UNIEDU, CNPq, PROAP/CAPES and FAPESC/UDESC/PAP for providing financial support for this research.

RESUMO: A integração lavoura-pecuária implica no equilíbrio do forrageamento adequado dos animais, minimizando os impactos nas áreas agrícolas graníferas. Os objetivos deste trabalho foram verificar, em trigo, os efeitos das alturas pré-corte $(20$ e $30 \mathrm{~cm}$ ) e do número de cortes (sem corte, 1,2 e 3 cortes) sobre a produtividade e composição bromatológica da forragem, e a produtividade de grãos dos cultivares BRS Umbu e BRS Tarumã. Independente do número de cortes, a altura de $30 \mathrm{~cm}$ proporcionou maior produtividade de forragem (1032 e $\left.1348 \mathrm{~kg} \mathrm{MS} \mathrm{ha}^{-1}\right)$ do que $20 \mathrm{~cm}$ (554 e $\left.909 \mathrm{~kg} \mathrm{MS} \mathrm{ha}^{-1}\right)$, para os cultivares BRS Umbu e BRS Tarumã, respectivamente, na média das safras. Para ambos cultivares e safras, obteve-se valores médios de $26 \%$ de proteína bruta, $55 \%$ de fibra em detergente neutro e $26 \%$ de fibra em detergente ácido. A $20 \mathrm{~cm}$ não foram encontradas diferenças na produtividade de grãos em função do número de cortes, com $3780 \mathrm{~kg} \mathrm{ha}^{-1}$ para BRS Umbu na média das safras e $4306 \mathrm{~kg} \mathrm{ha}^{-1}$ em 2014 para BRS Tarumã. Porém, para ambos os cultivares a $30 \mathrm{~cm}$, o rendimento de grãos foi prejudicado em $30 \%$ no terceiro corte. Estes resultados indicam que o trigo duplo-propósito pode ser empregado no sul do Brasil em sistemas de pastejo em épocas de escassez de forragem e ainda produzir grãos sem penalidades, de acordo com o manejo empregado. 
PALAVRAS-CHAVE: Triticum aestivum L. Sistema de integração lavoura pecuária. Altura de manejo. Número de cortes. Produção de grãos.

\section{REFERENCES}

Association of official analytical chemists - AOAC. 1990. Official methods of analysis. 15th ed. 1: 72-74p.

ARAÚJO, D. L. C.; OLIVEIRA, M. E.; LOPES, J. B.; ALVES A. A.; RODRIGUES, M. M.; MOURA, R. L.; SANTOS, M. S. Morphogenetic characteristics and demographic patterns of tillers on andropogon grass under different forage allowances. Semina: Ciências Agrárias, Londrina, v. 36, n. 5, p. 3303-3314, 2015. https://doi.org/10.5433/1679-0359.2015v36n5p3303

BARTHRAM, G. T. Experimental techniques: the HFRO sward stick. In: HFRO. The Hill Farming Research Organization Biennal Report 1984/1985. Penicuik: HFR, p. 29-30. 1985.

BELL, L. W.; HARRISON, M. T.; KIRKEGAARD, J. A. Dual-purpose cropping - capitalising on potential grain crop grazing to enhance mixed-farming profitability. Crop and Pasture Science, Australia, v. 66, p. 1-4, 2015. https://doi.org/10.1071/CPv66n4_FO

BORTOLINI, P. C.; SANDINI, I.; CARVALHO, P. C. F.; MORAES, A. Winter cereals submitted to cuts in double purpose system. Revista Brasileira de Zootecnia, Viçosa, v. 33, n. 1, p. 45-50, 2004.

https://doi.org/10.1590/S1516-35982004000100007

BUTCHEE, J. D.; EDWARDS, J. T. Dual-purpose wheat grain yield as affected by growth habit and simulated grazing intensity. Crop Science, Madison, v. 53, n. 4, p. 1686-1692, 2013.

https://doi.org/10.2135/cropsci2013.01.0033

CARLETTO, R., NEUMANN, M.; LEÃO, G. F. M.; HORST, E. H.; ASKEL, E. J. Effect of cut systems on production and quality of dual-purpose wheat grains. Revista Acadêmica: Ciência Animal, Curitiba, v. 13, n. 1, p. 125-133, 2015. https://doi.org/10.7213/academica.13.FC.AO13

EMBRAPA. Empresa Brasileira de Pesquisa Agropecuária. Sistema Brasileiro de Classificação de Solos. 3 ed. Brasília, 2013. 353 p. Available in:< https://livimagens.sct.embrapa.br/amostras/00053080.pdf $>$ Access in: August, 18, 2016.

FONTANELI, R. S.; SANTOS, H. P.; FONTANELI, R. S. (Eds.). Forrageiras para integração lavourapecuária-floresta na região sul-brasileira. Passo Fundo: Embrapa Trigo, 2012. 544p.

https://doi.org/10.1590/S1516-35982009001100007

FONTANELI, R.S. et al. Yield and nutritive value of dual purpose winter cereals: green forage, silage or grain. Revista Brasileira de Zootecnia, Vicosa, v. 38, n. 11, p. 2116-2120, 2009.

HARRISON, M. T.; EVANS, J. R.; DOVE, H.; MOORE, A. D. Dual-purpose cereals: Can the relative influences of management and environment on crop recovery and grain yield be dissected? Crop \& Pasture Science, Clayton South, v. 62, n. 11, p. 930-946, 2011. https://doi.org/10.1071/CP11066

HARRISON, M. T.; KELMAN, W. M.; VIRGONA, J. M. Effects of grazing on crop crown temperature: Implications for phenology. Crop and Pasture Science, Clayton South, v. 66, n. 4, p. 235-248, 2014. https://doi.org/10.1071/CP13380 
HASTENPFLUG, M.; BRAIDA, J. A.; MARTIN, T. N.; ZIECH, M. F.; SIMIONATTO, C. C.; CASTAGNINO, D. S. Dual-purpose wheat cultivars submitted to nitrogen fertilization and cutting regimes. Arquivo Brasileiro de Medicina Veterinária e Zootecnia, Belo Horizonte, v. 63, n. 1, p. 196-202, 2011 a. https://doi.org/10.1590/S0102-09352011000100029

HASTENPFLUG, M.; MARTIN, T. N.; BRAIDA, J. A.; BARBOSA, D. K.; ZIELINSK, R. P.; REFATT, R. Grain yield of dual-purpose wheat cultivars as affected by nitrogen and cuttings. Bragantia, Campinas, v. 70, n. 4, p. 819-824, 2011b. https://doi.org/10.1590/S0006-87052011000400013

HENDRICKSON, J. R.; BERDAHL, J. D.; LIEBIG, M. A.; KARN, J. F. Tiller persistence of eight intermediate wheatgrass entries grazed at three morphological stages. Agronomy Journal, Madison, v. 97, $\mathrm{n}$. 5, p. 1390-1395, 2005. https://doi.org/10.2134/agronj2004.0179

HENZ, É.L.; DE ALMEIDA, P. S. G.; VELHO, J. P.; NÖRNBERG, J. L.; SILVA, L. D. F.; BACKES, T. R.; GUERRA, G. L. Dual purpose wheat production with different levels of nitrogen topdressing. Semina:

Ciências Agrárias, Londrina, v. 37, n. 2, p 1091-1100, 2016. https://doi.org/10.5433/16790359.2016v37n2p1091

HODGSON, J. Grazing management: science into practice. New Zealand: Longman Scientific \& Technical, 1990. $204 \mathrm{p}$.

ISLAM, M.; ANWAR, S.; BASHIR, S.; KHATTAK, W.A.; IMRAN, I.; ALI, M.; KHAN, N. Growth and yield components of wheat varieties as affected by dual purpose practices. Pure and Applied Biology, Bolan, v. 4, n. 4, p. 491-496, 2015. https://doi.org/10.19045/bspab.2015.44006

LEHMEN, R. I.; FONTANELI, R. S.; FONTANELI, R. S.; SANTOS, H. P. Yield, nutritive value and fermentative parameters of winter cereals silages. Ciência Rural, Santa Maria, v. 44, n. 7, p. 1180-1185, 2014. https://doi.org/10.1590/0103-8478cr20130840

LI, P. F.; MA, B. M.; YAN, W.; CHENG, Z. G.; LI, F. M.; XIONG, Y. C. Plant architecture, plasticity, and adaptation strategies of two oat genotypes under different competition intensities. Journal of the Science of Food and Agriculture, New York, v. 96, n. 5, p. 1431-1439, 2016. https://doi.org/10.1002/jsfa.7237

MACKOWN, C. T.; CARVER, B. F. Fall forage biomass and nitrogen composition of winter wheat populations selected from grain - only and dual-purpose environments. Crop Science, Madison, v. 45, n. 1, p. 322-328, 2005. https://doi.org/10.2135/cropsci2005.0322

MARIANI, F.; FONTANELI, R. S.; VARGAS, L.; SANTOS, H. P.; FONTANELI, R. S. Double purpose wheat and oat pasture evaluation after perennial forage and summer crops in croplivestock systems. Ciência Rural, Santa Maria, v. 42, n. 10, p. 1752-1757, 2012. https://doi.org/10.1590/S0103-84782012001000006

MARTIN, T. N.; SIMIONATTO, C . C.; BERTONCELLI, P.; ORTIZ, S.; HASTENPFLUG, M.; ZIECH, M. F.; SOARES, A. B. Phytomorphology and production of dual purpose wheat with different cutting regimes and seeding density. Ciência Rural, Santa Maria, v. 40, n. 8, p. 1695-1701, 2010. https://doi.org/10.1590/S010384782010000800004

MARTIN, T. N.; STORCK, L.; BENIN, G.; SIMIONATTO, C. C.; ORTIZ, S.; BERTONCELLI, P. Importance of the relationship between characters in dual purpose wheat in crop breeding. Bioscience Journal, Uberlândia, v. 29, n. 6, p. 1932-1940, 2013.

MATTHEW, C.; LEMAIRE, G.; SACKVILLE HAMILTON, N.R.; HERNANDEZ-GARAY, A. A modified self-thinning equation to describe size/density relationships for defoliated swards. Annals of Botany, Oxford, v. 76, n. 6, p. 579-587, 1995. https://doi.org/10.1006/anbo.1995.1135 
MEINERZ, G. R.; OLIVO, C. J.; FONTANELI, R. S.; AGNOLIN, C. A.; FONTANELI, R. S.; HORST, T.; VIÉGAS, J.; DE BEM, C. M. Nutritive value of forage of genotypes of double purpose winter cereals. Revista Brasileira de Zootecnia, Viçosa, v. 40, n. 6, p. 1173-1180, 2011. https://doi.org/10.1590/S151635982011000600003

MEINERZ, G. R.; OLIVO, C. J.; FONTANELI, R. S.; AGNOLIN, C. A.; HORST, T.; DE BEM, C. M. Productivity of double-purpose winter cereals in the Depressão Central region of Rio Grande do Sul state. Revista Brasileira de Zootecnia, Viçosa, v. 41, n. 4, p. 873-882, 2012. https://doi.org/10.1590/S151635982012000400007

MEZZALIRA, J. C.; CARVALHO, P. C. F.; FONSECA, L.; BREMM, C.; CANGIANO, C.; GONDA, H. L.; LACA, E. A. Behavioural mechanisms of intake rate by heifers grazing swards of contrasting structures.

Applied Animal Behaviour Science, Oxford, v. 153, n. 1, p. 1-9, 2014.

https://doi.org/10.1016/j.applanim.2013.12.014

NAVEED, K.; KHAN, M. A.; BALOCH, M. S.; ALI, K.; NADIM, M. A.; KHAN, E. A.; SHAH, S.; ARIF, M. Effect of different seeding rates on yield attributes of dual-purpose wheat. Sarhad Journal of Agriculture, Peshawar, v. 30, n. 1, p. 83-91, 2014.

NAVEED, K.; KHAN, M. A.; BALOCH, M. S.; ARIF, M.; NAQVI, S. A.; KHAN, J.; ALI, S. Early planting date can compensate the reduction in wheat yield due to fodder cutting in dual purpose wheat. Pakistan Journal of Agricultural Sciences, Faisalabad, v. 52, n. 2, p. 469-477, 2015.

National Research Council- NRC. 2001. Nutrient requirements of dairy cattle. 7.ed. rev. Washington, DC: National Academy Press. 408 p.

PEREIRA, M. J. R.; BONAN, E. C. B.; GARCIA, A.; VASCONCELOS, R. L.; GÍACOMO, K. S.; LIMA, M. F. Morphoagronomic characteristics of maize in response to different levels of defoliation. Revista Ceres, Vicosa, v. 59, n. 2, p 200-205, 2012. http://dx.doi.org/10.1590/S0034-737X2012000200008

RAMELLA, J. R. P.; LIBARDI, K. D. C.; CASTAGNARA, D. C.; MOTTIN, M. C.; SEIDEL, E. P.; OLIVEIRA, P. S. R. Agricultural yield components of dual purpose wheat cv. BRS Tarumã under cutting and nitrogen fertilization handlings. African Journal of Agricultural Research, Nairobi, v. 10, n. 8, p. 811-820, 2015. https://doi.org/10.5897/AJAR2014.9166

SAKAMOTO, T.; MORINAKA, Y.; OHNISHI, T.; SUNOHARA, H.; FUJIOKA, S.; UEGUCHI-TANAKA, M.; MIZUTANI, M.; SAKATA, K.; TAKATSUTO, S.; YOSHIDA, S.; TANAKA, H.; KITANO, H.;

MATSUOKA, M. Erect leaves caused by brassinosteroid deficiency increase biomass production and grain yield in rice. Nature Biotechnology, Basingstoke, v. 24, n. 1, p. 105-109, 2006.

http://dx.doi.org/10.1038/nbt1173

SANGOI, L.; BERNS, A. C.; ALMEIDA, M. L.; ZANIN, C. G.; SCHWEITZER, C. Agronomic characteristics of wheat cultivars in response to the time of nitrogen fertilizer covering. Ciência Rural, Santa Maria, v. 37, n. 6, p. 1564-1570, 2007. http://dx.doi.org/10.1590/S0103-84782007000600010

SANTOS, H. P.; FONTANELI, R. S.; CASTRO, R. L. DE; VERDI, A. C.; VARGAS, A. M.; BIAZUS, V. Available of wheat cultivated for grain production and for dual-purpose under no-tillage. Revista Brasileira de Ciências Agrárias (Agrária), Recife, v. 10, n. 1, p. 43-48, 2015. https://doi.org/10.5039/agraria.v10i1a4567

SEYMOUR, M.; ENGLAND, J. H.; MALIK, R.; ROGERS, D.; SUTHERLAND, A.; RANDELL, A. Effect of timing and height of defoliation on the grain yield of barley, wheat, oats and canola in Western Australia. Crop and Pasture Science, Clayton South, v. 66, n. 4, p. 287-300, 2015. https://doi.org/10.1071/CP13411 
SOARES, A. B.; PIN, E. A.; POSSENTI, J. C. Nutritive value of five cool-season forage species under four sowing dates. Ciência Rural, Santa Maria, v. 43, n 1, p. 120-125, 2013. http://dx.doi.org/10.1590/S010384782012005000131

TAFFAREL, L. E.; OLIVEIRA, P. S. R.; OLIVEIRA, E. R.; MUNIZ, E. B.; MESQUITA, E. E.; COSTA, P. B.; PIANO, J. T.; NERES, M. A.; COSTA, P. F. Morphological characteristics, dry matter production, and nutritional value of winter forage and grains under grazing and split nitrogen fertilization. Semina: Ciências Agrárias, Londrina, v. 38, n. 3, p. 1473-1490, 2017. http://dx.doi.org/10.5433/1679-0359.2017v38n3p1483

TIAN, L.H.; BELL, L. W.; SHEN, Y. Y.; WHISH, J. P. M. Dual-purpose use of winter wheat in western China: Cutting time and nitrogen application effects on phenology, forage production, and grain yield. Crop and Pasture Science, Clayton South, v. 63, n. 6, p. 520-528, 2012. https://doi.org/10.1071/CP12101

TONETTO, C. J.; MÜLLER, L.; MEDEIROS, S. L. P.; MANFRON, P. A.; BANDEIRA, A. H.; MORAIS, K. P.; LEAL, L. T.; MILTTEMANN, A.; NETO, D. D. Production and chemical composition of diploid and tetraploid genotypes of ryegrass. Zootecnia Tropical, Venezuela, v. 29, n. 2, p. 169-178, 2011.

VAN SOEST, P. J. Symposium on factors influencing the voluntary intake of herbage by ruminants: voluntary intake relation to chemical composition and digestibility. Journal of Animal Science, Oxford, v. 24, n. 3, p. 834-844, 1965.

VAN SOEST, P. J., ROBERTSON, J. B.; LEWIS, B. A. Methods for dietary fiber, neutral detergent and nonstarch polysaccharides in relation to animal nutrition. Journal of Dairy Science, Amsterdam, v. 74, n. 10, p. 3583-3597, 1991.

VIRGONA, J. M.; GUMMER, F. A. J.; ANGUS, J. F. Effects of grazing on wheat growth, yield, development, water use, and nitrogen use. Australian Journal of Agricultural Research, Clayton Austrália, v. 57, n. 12, p. 1307-1319, 2006.

YODA, K.; KIRA, T.; OGAWA, H.; HOZUMI, K. Self-thinning in overcrowded pure stands under cultivated and natural conditions. Journal of Biology, Osaka City University, Osaka, v. 14, n. 1, p. 107-129, 1963.

ZADOKS, J. C.; CHANG, T. T.; KONZAK, C. F. A decimal code for the growth stages of cereals. Weed Research, Oxford, v. 14, n. 6, p. 415-421, 1974. http://dx.doi.org/10.1111/j.1365-3180.1974.tb01084.x.

ZANON, A. J.; STRECK, N. A.; ROSA, H. T.; WALTER, L. C.; ALBERTO, C. M. Leaf number associated with double ridge and terminal spikelet in wheat cultivars. Revista Ciência Agronômica, Fortaleza, v. 43, n. 3, p, 569-578, 2012. https://doi.org/10.1590/S1806-66902012000300021 